\title{
EVOLUCION JURÍDICA DEL VÍNCULO BIOLÓGICO EN LA MATERNIDAD: HACIA LA RUPTURA
}

\author{
LEGAL EVOLUTION OF BIOLOGICAL LINK IN THE \\ MOTHERHOOD: TOWARDS THE RUPTURE
}

\section{EVOLUÇÃO LEGAL DO VÍNCULO BIOLÓGICO NA MATERNIDADE: RUMO À RUPTURA}

Frédéric Mertens de Wilmars ${ }^{1^{*}}$

\begin{abstract}
1 Introducción. 2 Del omnsiciente vínculo biológico en la maternidad. 2.1 El control político de la maternidad. 2.2 La maternidad biológica consagrada por el Código Civil. 3 A la pluralidad de maternidades. 3.1 Un vínculo biológico y dos estatus de madre. 3.2 La libertad de elegir la (no) maternidad. 3.3 La singularidad de la maternidad: del hecho biológico a la intención de maternidad. 4 La gestación subrogada: transición para la ruptura definitiva del vínculo biológico en la maternidad. 5 Conclusiones. Referências.
\end{abstract}

\section{RESUMEN}

Los debates actuales relativos a la gestación subrogada se inscriben en la perspectiva de la ruptura del vínculo biológico en la maternidad. La ciencia aleja siempre más los límites a la procreación. A través del Derecho, el Estado trata de controlar el desarrollo de esta técnica que replantea las cuestiones jurídicas de la filiación materna. La maternidad biológica siempre ha sido considerada como única por motivos jurídicos y políticos. Ahora bien, la ciencia y el Derecho han permitido la emergencia de otros tipos de maternidad. Además, las técnicas reproductivas, la gestación subrogada, así como la inminencia de la ectogénesis conducen a la ruptura jurídica del vínculo biológico de la maternidad, puesto que ésta es posible en un contexto de cambio sociológico que hace prevalecer la libre elección de la (no)maternidad y la igualdad de género ante el hecho de la Naturaleza.

Palabras clave: Filiación biológica. Igualdad. Maternidad múltiple. Gestación subrogada. Ectogénesis.

1 Doctor en Derecho Constitucional y Profesor en la Universidad Europea de Valencia (España). E-mail: <frederic. mertensdewilmars@universidadeuropea.es>. http://orcid.org/0000-0002-4198-9017 


\begin{abstract}
Current debates regarding surrogacy are inscribed in the perspective of the breakdown of the biological link in motherhood. Science always pushes the limits to procreation further. Through the rules of Law, the State tries to control the development of this technique that rethinks the legal issues of maternal affiliation. Biological motherhood has always been considered as unique for legal and political reasons. Now, Science and Law have allowed the emergence of other types of motherhood. In addition, reproductive techniques, surrogacy, as well as the imminence of ectogenesis lead to the legal breakdown of the biological link of motherhood, since this is possible in a context of sociological change that privileges the free choice of (no-) maternity and gender equality in the face of Nature.
\end{abstract}

Keywords: Biological Affiliation. Equality. Multiple Maternity. Surrogate Pregnancy. Ectogenesis.

\title{
RESUMO
}

Os debates atuais sobre barriga de aluguel estão inscritos na perspectiva da quebra do vínculo biológico na maternidade. A evolução da ciência cada vez mais amplia os limites para a procriação. Por meio da lei, o Estado tenta regular as repercussões legais da evolução de tais técnicas da filiação materna. A maternidade biológica sempre foi considerada única por razões legais e políticas. Agora, Ciência e Direito permitiram o surgimento de outros tipos de maternidade. Além disso, técnicas reprodutivas, barriga de aluguel e a iminência da ectogênese levam ao colapso legal do vínculo biológico da maternidade, uma vez que isso é possível em um contexto de mudança sociológica que privilegia a livre escolha de (não) maternidade e gênero. igualdade diante da natureza.

Palavras-chave: Afiliação Biológica. Igualdade. Maternidade Múltipla. Gravidez Substituta. Ectogênese.

\section{INTRODUCCIÓN}

En el Derecho, la determinación de la madre biológica es obvia. "Mater Semper certa est". La maternidad renvía, ante todo y exclusivamente, a un proceso biológico que consiste en la gestación necesaria al nacimiento de un ser humano, del cual derivan consecuencias jurídicas y políticas. Frente a la evidencia de la maternidad biológica, desde la Antigüedad hasta ahora, el Estado ha tomado rápidamente su control para definir la filiación materna y sus modos de establecimiento, persiguiendo así objetivos jurídicos - de índole privado, como el Derecho privado - y fines políticos - la cohesión social y el control demográfico.

Los cambios en el "arte de fabricar bebés" que se han producido en las últimas tres décadas a través del uso de la reproducción asistida han llevado a la creación de una nueva articulación entre la procreación y la filiación. Al mismo tiempo, generaron cambios importantes en las representaciones de la maternidad. Por circunstancias de la Naturaleza o una cuestión de libre elección - las ideas y las costumbres evolucionando respecto a la (no)maternidad - las variantes 
de la maternidad se han fragmentado de acuerdo con las diferentes técnicas de reproducción utilizadas, las diversas culturas de filiación en las que encajan, así como a través de los Estados y sus sistemas legislativos respectivos que las enmarcan o prohíben.

La maternidad ya no es exclusivamente biológica. Puede disociarse en diversas figuras de maternidad. Así pues, una mujer puede haber deseado a un hijo o hija gestado(a) y nacid(o)a de otra mujer, y educad(o)a por una tercera mujer. Una cuarta mujer podría también proveer el material genético necesario para la procreación. Cuatro mujeres tienen, en definitiva, un papel variante de maternidad. Dos de ellas ejercen una maternidad basada en la voluntad - la mujer que desea al niño y la que lo educa -, otra la ejerce en base a un embarazo y, finalmente, la cuarta ejerce una maternidad fundada en la genética.

Desde la perspectiva jurídica, se plantea la cuestión de determinar si se trata de una misma maternidad bajo varias dimensiones o más bien de una pluralidad de maternidades, cada una siendo regida por una normativa específica. Ahora bien, esas maternidades deben tratarse como distintas. En efecto, si es naturalmente concebible que la mujer que porta un niño y le pare constituye el modelo de representación de la maternidad, limitarse a esta figura materna excluye a las otras mujeres que podrían reivindicarse como madres. En otros términos, entre todas esas maternidades, haría que determinar la(s) que debe(n) tener una base jurídica.

Esta cuestión reviste hoy una acuidad peculiar con la gestación subrogada porque replantea el marco jurídico del vínculo biológico en la maternidad. De hecho, esta técnica reproductiva desvincula la maternidad de la automatización de la relación filial, en una transición provisional hacia la procreación del ser humano sin la intervención corporal de una mujer.

Así pues, la presente contribución analiza la evolución de la maternidad basada en un vínculo biológico que tradicionalmente era la vía principal para establecer la filiación materna. Con el cambio del estatuto de la mujer en la sociedad y las posibilidades para ella de elegir o no la maternidad, la relación biológica entre la mujer y la hija o el hijo ya no es la base fundamental de la maternidad. En este sentido, la gestación subrogada es el paradigma de una nueva maternidad radicada en la intención o la voluntad de la mujer.

En una primera parte, destacamos el papel del Derecho en el establecimiento de la maternidad biológica, así como el del Estado en el control de sus modalidades. La segunda parte toma en consideración la emergente pluralidad de maternidades en un contexto jurídico, político y sociocultural que cuestiona la exclusividad del vínculo biológico en la maternidad. Finalmente, en una tercera parte, se subrayan las incidencias de la gestión subrogada sobre la evolución jurídica del vínculo biológico en la maternidad hacia una ruptura definitiva.

\section{DEL OMNSICIENTE VÍNCULO BIOLÓGICO EN LA MATERNIDAD}

Debido a su importancia para la supervivencia de cualquier sociedad, la maternidad siempre ha sido controlada por el poder y enmarcada por el Derecho. Las razones políticas y jurídicas se centran en la reproducción de niñas y niños que tiene incidencias tanto en las 
relaciones entre los individuos - el Derecho Privado - como en las relaciones de éstos con el Estado - el Derecho Público.

\subsection{EL CONTROL POLÍTICO DE LA MATERNIDAD}

A lo largo de la Historia, la mayoría de los Estados ha desarrollado políticas natalistas porque la natalidad es la clave para el poder político. La demografía y su control en términos de gestión se perciben como un medio básico de crecimiento de la potencia económica y militar de una nación ${ }^{2}$.

La maternidad representa una cuestión fundamental para la sociedad en su dimensión pública y el poder - democrático o no - genera una cierta valorización, véase una glorificación del rol materno, sean cuales sean la época y el lugar porque la madre es el "pivote central" de la familia y de la sociedad.

Además, el Estado enmarca la maternidad porque ésta también constituye un elemento de identificación del niño o la niña y consecuentemente del individuo en la sociedad. El hecho materno del parto confiere al niño o la niña el estatuto de sujeto de derecho, y le permite anclarse en una célula familiar a través del reconocimiento de una filiación que genera derechos y obligaciones. El estado jurídico del niño o de la niña se determina a su nacimiento, cuando está físicamente desvinculado(a) de su madre por el corte del cordón umbilical.

Ahora bien, mientras que la "maternidad" evoca el vínculo de la madre con la niña o el niño desde el punto de vista de la madre, la expresión de "filiación materna" se sitúa desde el punto de vista del niño o la niña. Ambas expresiones participan, sin embargo, a una misma idea de vinculación jurídica entre la niña o el niño y la mujer a quienes les ha dado luz. En la medida en que la maternidad es determinante para la identificación del niño o la niña, es el Estado que fija los criterios de establecimiento del vínculo filial.

En la mayoría de las sociedades pasadas y actuales, el vínculo jurídico del niño o la niña con la madre y el proceso biológico materno están correlacionados. Esta correlación se debe a la certidumbre del proceso biológico materno: "Mater Semper certa est". En otras palabras, la maternidad aparece como un hecho natural: el increíble poder de una mujer para crear a una niña o un niño podía hacer creer que convertirse en una madre era una evidencia (FONTY; HUGUENIN, 2003). El poder político y el Estado lo han entendido así; lo que explica la involucración del legislador y el Derecho en el establecimiento de la filiación materna.

\subsection{LA MATERNIDAD BIOLÓGICA CONSAGRADA POR EL CÓDIGO CIVIL}

El nacimiento materializa una fuente de efectos jurídicos, como el de tener una personalidad en el sentido del Derecho. El artículo 30 del Código Civil español indica que "la personalidad se adquiere en el momento del nacimiento con vida, una vez producido el entero

2 Por ejemplo, después de cuarenta años de "política del hijo único", China está elaborando en un nuevo Código Civil que ya no debería hacer ninguna referencia al "control de la natalidad". Después de su adopción en 2020 por la Asamblea Nacional, todas las parejas chinas podrán elegir el número de hijos e hijas que desean. Según los demógrafos, China claramente se lanzará a una política natalista (ROWLAND, 2015). 
desprendimiento del seno materno.” (ESPAÑA, 1889, p. 22). Así pues, el Derecho Civil exige que la persona tenga una madre y que haya un vínculo biológico que, paradójicamente, se manifiesta a través de una separación física entre la madre y la niña o el niño. Es entonces la maternidad biológica que determina el estatuto del niño o de la niña en el marco del vínculo jurídico de éstos con la madre.

Tanto para la sociedad como para el Derecho, la maternidad siempre ha sido percibida como un hecho de la Naturaleza, intemporal, universal, como un componente esencial y banal de la identidad femenina. Esta concepción de la maternidad ha sido la base de una visión tradicional y profundamente desigualitaria de la familia en el seno de la cual la mujer tiene una plaza restringida debido a su estatuto.

Al elegir la correlación entre le hecho biológico y la filiación, el Derecho ha cristalizado la intangibilidad del estado civil. La mujer que da luz identifica al niño o la niña en la sociedad. La importancia de la determinación de la madre biológica en el ámbito jurídico aparece entonces.

La ley de la Naturaleza se confunde con la ley de las mujeres y de los hombres. Esta confusión es tal que no merece la pena de ser examinada a la luz del Derecho. Por ello, el legislador y la doctrina no han desarrollado numerosas disposiciones relativas a la maternidad, aunque ésta nunca ha sido totalmente ausente de los textos jurídicos ${ }^{3}$.

Con el tiempo, han sido publicados textos que tratan de la maternidad, pero cuantitativa y cualitativamente siguen siendo menos importantes que las publicaciones relativas a la paternidad. De hecho, es sólo a partir de los años 1980 que el sistema legal español empezó a integrar normas realmente dedicadas a la maternidad, incorporando el principio de igualdad entre hombres y mujeres a través de las directivas europeas sobre la protección de las trabajadoras embarazadas y el permiso parental ${ }^{4}$.

En definitiva, el Derecho limita la maternidad a la reproducción al simple hecho biológico de la concepción. Por este motivo, hay autores que diferencian la maternidad de la madre (MARRADES PUIG, 2002). Para el Derecho, la maternidad está vinculada con el periodo de embarazo, parto y lactancia mientras que el concepto de madre es más amplio y largo en el tiempo porque incluye elementos no biológicos que forman un construido social basa en una relación materno-filial (GUERRINA, 2005).

\section{A LA PLURALIDAD DE MATERNIDADES}

3 En cambio, la paternidad ha sido objeto de muchas normas jurídicas por el motivo de su indeterminación físi$\mathrm{ca}$ - aunque, desde hace varios años existe el recurso a la técnica de ADN en la prueba de filiación paterna. El Código Civil español actual refleja aun la predominancia de la paternidad sobre la maternidad, las disposiciones relativas a la filiación siendo estructuradas bajo el título V "De la paternidad y filiación”. En su conjunto, el Código es desigualitario puesto que menos de una decena de disposiciones evocan la maternidad y, además sólo treinta y seis veces menciona el término de "madre" mientras que el de "padre" aparece ciento veintiséis.

4 Reenviamos, por ejemplo, a la Ley Orgánica 3/2007, de 22 de marzo, para la igualdad efectiva de mujeres y hombres que menciona la maternidad preconizando la necesidad de asumir sus costos sociales colectivos. 
La dicotomía de los papeles entre mujer y hombre en la creación de la filiación se ha trasladado sobre el estatuto de la mujer y de la mujer casada, en la medida en que, si en los hechos, la carga de la educación incumbía en prioridad a la mujer, jurídicamente ésta era "ocultada" por ser casada. Paradójicamente, la madre soltera, llamada "madre natural", tenía un estatuto más visible que la "mujer legítima" - la mujer casada. Ya dos tipos de madre estaban contemplados por el Derecho.

Sin embargo, la "(r)evolución" sociocultural de las ideas con "Mayo de 1968" y la revolución sexual de los años 1960 (MIGUEL ÁLVAREZ, 2015), han conducido la maternidad a sobrepasar el vínculo biológico porque, a partir de entonces, la mujer tenía la posibilidad de elegir ser madre o no.

Esta posibilidad revolucionaria de cambio de estatuto sociocultural para la mujer, al no ser automáticamente convertible en madre, ha contribuido a la redefinición de la maternidad porque ésta ya no exclusivamente biológica: es múltiple. En otras palabras, tanto la sociedad como el Derecho subrayan la singularidad de la maternidad que radica en la voluntad de ser madre con el apoyo - si necesario - del progreso científico.

\subsection{UN VÍNCULO BIOLÓGICO Y DOS ESTATUS DE MADRE}

Hasta hace poco, la mujer no tenía las mismas capacidades ni los mismos derechos que el hombre, sobre todo cuando ella estaba casada. Si el Derecho afirmaba la inferioridad de la mujer respecto al hombre, en cambio, la función femenina materna era valorizada en el seno de la unión matrimonial.

La situación de la mujer casada se opone entonces a la de la mujer non casada, debido al rechazo - sino social, al menos jurídico - de las uniones libres. De hecho, tradicionalmente, el Derecho ha enfocado la familia como una concepción matrimonial.

Así pues, la Declaración Universal de los Derechos Humanos de 1948 (en adelante, DUDH) en su artículo 16, reconoce el derecho a casarse y a tener una filiación ${ }^{5}$. Resulta significativo que la Declaración conecte el derecho a crear una filiación con el derecho a casarse. Esta conexión ha planteado la cuestión del matrimonio como única forma legítima de crear una filiación. Generalmente, la doctrina internacional ha considerado que el matrimonio es la institución que favorece y facilita la natalidad y el crecimiento de la prole.

La formulación de la disposición precitada del DUDH, contrasta claramente con la situación jurídica actual de los Estados occidentales, en la cual, cada vez más, se reconoce que el matrimonio no es la única forma de constituir una filiación, abriendo el derecho a nuevos modelos de familia y de filiación. La divisibilidad de la filiación fuera del matrimonio ha generado el hecho que la filiación materna puede establecerse sin que el vínculo de la filiación paterna sea determinado.

Desde un punto de vista jurídico, la madre "natural” podía ser única progenitora de

5 Naciones Unidas (1948): Declaración Universal de los Derechos Humanos. Adoptada y proclamada por la Asamblea General en su Resolución 217 A (iii), de 10 de diciembre de 1948. 
un niño o una niña nacido(a) fuera del matrimonio, al contrario de la madre legítima. En consecuencia, la filiación materna natural producía efectos más amplios que la descendencia materna legítima, porque la madre natural no estaba bajo la autoridad de un marido.

Hasta mediados del siglo XX, la familia estaba regida por un modelo patriarcal y matrimonial que ha sido la base de una situación desigual de las mujeres como madres. Esta desigualdad de los sexos y de las parejas necesariamente tuvo consecuencias sobre los efectos jurídicos de los vínculos de la filiación, puesto que ésta figuraba en el centro de la familia. Tratadas de manera distinta respecto a los padres, las madres también eran tratadas de manera distinta entre ellas con la discriminación entre las madres naturales y las madres legitimas.

El diseño de maternidad fue, por lo tanto, singular en la medida en que, si la madre biológica apareció como la figura cierta, finalmente había dos diferentes regímenes de maternidad: un régimen legítimo de maternidad y un régimen de maternidad natural. Así pues, no había una sino varias maternidades.

\subsection{LA LIBERTAD DE ELEGIR LA (NO) MATERNIDAD}

Desde los años 1960, las mujeres han conseguido liberarse de las restricciones vinculadas a la maternidad para poder realizarse como individuos en la sociedad. Una revolución copernicana entonces ha cambiado la concepción de la sociedad sobre la maternidad y la condición femenina. Con la incorporación masiva de las mujeres en el mercado laboral, el papel de la mujer en la sociedad se ha diversificado. La mujer ya no es sólo una madre. Tiene proyectos otros que sólo tener hijos o hijas.

Con el progreso científico, la mujer tiene derecho a rechazar la maternidad, provisional o definitivamente, tanto biológica como jurídicamente. La contracepción le permite controlar su fecundidad y ella puede recurrir al aborto que se ha constituido en un verdadero derecho a partir de los años 1970. Además, en algunos países, la mujer que decide dar a luz puede hacerlo de manera anónima ${ }^{6}$ y rechazar ser madre desde el punto de vista jurídico al refutar el establecimiento del vínculo jurídico con la niña o el niño.

Así pues, la mujer tiene la posibilidad de disociar jurídicamente la filiación materna voluntaria de la filiación biológica. Al reconocerle el dominio de su capacidad a tener hijas e hijos como prerrogativa femenina, el Derecho otorga a la mujer - soltera o no - la facultad de convertir una maternidad impuesta en una maternidad deseada.

La familia ha evolucionado hacia un modelo igualitario, véase femenino. La maternidad sigue siendo la prerrogativa de las mujeres. Hoy en día, el reconocimiento del control biológico y jurídico de la maternidad conduce a conferir una creciente importancia a la madre, a su elección, a su intención. En otras palabras, la elección de tener a una hija o un hijo incumbe hoy a la mujer.

6 Por ejemplo, en Francia, Luxemburgo e Italia, existe el parto en el anonimato. Hasta 1999, una mujer española podía dar a luz de manera anónima sólo en el caso de ser soltera. En su sentencia STS 776/1999, del 21 de septiembre, el Tribunal Supremo suprimió esta posibilidad. 


\subsection{LA SINGULARIDAD DE LA MATERNIDAD: DEL HECHO BIOLÓGICO A LA INTENCIÓN DE MATERNIDAD}

La singularidad del fenómeno materno implica interrogarse sobre la vinculación de la hija o el hijo con una mujer que toma el estatuto de madre, así como los efectos de esta vinculación en la perspectiva de la maternidad. Al mismo tiempo, la paternidad no puede ser totalmente ocultada, en la medida en que la procreación se basa aún en la complementariedad de los sexos. Entonces, el niño o la niña siempre provienen de un hombre y de una mujer, al menos en plan genético; lo que cuestiona el reparto de los papeles entre el padre y la madre.

En realidad, el progreso técnico ha convertido el deseo utópico de hijo o hija de la mujer que estaba en incapacidad de ser madre. Ha hecho "nacer" la idea de que existe un derecho al niño o a la niña. Cualquier mujer es titular de un derecho subjetivo a ser madre. Se presenta entonces la cuestión de saber cómo el Derecho puede o debe reaccionar frente a las nuevas prácticas para tener hijos e hijas (GIAMI; PY, 2019).

Hoy, la maternidad es entonces lejos de ser una realidad simple La relación entre la maternidad en el sentido del hecho y la maternidad en el sentido jurídico se ha profundamente transformado no sólo por el motivo del reconocimiento del papel de las mujeres en la sociedad, sino también debido a la aparición de la pluralidad de maternidades.

Respecto a estos cambios, se cuestiona la relación entre maternidad de hecho y maternidad jurídica. La maternidad de hecho ha evolucionado. No sólo se trata de la gestación de un niño o una niña. Más ampliamente, es el hecho para una mujer de considerarse como madre de un hijo o una hija.

Esta evolución conceptual parece renovar la noción jurídica misma de la maternidad, tanto en sus efectos como en sus modalidades de establecimiento. ¿Qué es la figura materna y cuál es el papel para la madre? La evolución del Derecho de la familia ha reformado la relación del Derecho con la madre que, definitivamente, ya no radica exclusivamente en la figura tradicional de la maternidad gestacional.

En otros términos, si el embarazo y el parto son los elementos intangibles de la natalidad, la aparición de otras figuras maternas, basadas en el deseo o la intencionalidad de la mujer, conduce a la cuestión de la situación jurídica de cada una de éstas. La respuesta se basa en una cuestión de poder, y más precisamente en el poder de la voluntad con dos preguntas abiertas: ¿la voluntad es capaz de conferir a una mujer la cualidad jurídica de madre? ¿El control estatal y social de la maternidad puede retroceder hasta el punto de dejar el subjetivismo de la mujer determinar su estatuto y el de su hija o su hijo? (AGACINSKI, 2013).

\section{LA GESTACIÓN SUBROGADA: TRANSICIÓN PARA LA RUPTURA DEFINITIVA DEL VÍNCULO BIOLÓGICO EN LA MATERNIDAD}

La medicina ha progresado mucho en el ámbito de la procreación. Las técnicas de asis- 
tencia a la procreación llevan así nuevas soluciones a las dificultades para tener a una hija o un hijo y a la imposible maternidad.

La procreación puede ser asistida y la reproducción no se basa más “ipso facto” en una relación sexual entre una mujer y un hombre. La intervención médica puede responder a las infertilidades patológicas y también a las consecuencias del paso del tiempo para las mujeres. Además, la ciencia puede ser una alternativa para concebir niñas y niños, fuera de la vida de una pareja, véase fuera de una vida de pareja fundada en la alteridad sexual - hombre y mujer. La revolución científica radica, de hecho, en la disociación entre nacimiento y sexualidad.

La técnica ha desplazado los límites de la procreación natural, los frenos biológicos al nacimiento. Ha permitido a los seres humanos vencer la Naturaleza porque la medicina consigue "fabricarlos" sin ella. Sólo el núcleo duro del nacimiento constituido por la complementariedad de los sexos y la maternidad perdura. En otros términos, a pesar de la medicalización creciente de la procreación, las niñas y los niños deben siempre ser el resultado del encuentro entre los gametos masculinos y femeninos, y ser llevados - de momento - al mundo por las mujeres.

La disociación de la maternidad en varias figuras alcanza así su paroxismo porque la medicina puede hacer intervenir dos o tres mujeres en el proceso biológico de la natalidad: una mujer se encarga del embarazo y otra provee ovocitos. Así pues, una vez que la medicina integre este ámbito, las relaciones maternas establecidas en torno al niño o la niña llegan a ser más complejas. Ya no se limitan a la simple maternidad. La mujer que quiere ser madre ya no necesita ser capaz de dar a luz, puede delegar esta función a una tercera persona y externalizar así el proceso biológico del nacimiento. En definitiva, los limites al nacimiento no resultan más de la biología sino del Derecho (LEMOULAND, 2004).

Aunque tiene aún una dimensión marginal en comparación con otras técnicas de procreación asistida, la gestación subrogada genera tensiones entre lo posible - lo que la ciencia puede realizar - y lo que la sociedad considera como legítimo o no (BAUDOIN; LABRUSSE-RIOU, 1987).

Este procedimiento de procreación constituye un paso más hacia la ruptura definitiva del vínculo biológica en la maternidad. Si el Derecho ha contribuido paulatinamente a la emancipación de las mujeres y de los hombres respecto a la Naturaleza y la familia tradicional, la gestación subrogada implica no sólo la cuestión de su integración en el Derecho de la filiación sino la filiación misma que, por realidad o por ficción, está aún basada en un vínculo biológico (CANTO-SPERBER; FRYDMAN, 2008).

En la gestación subrogada, la madre ya no es la que da a luz. En una adopción, la madre adoptiva, social y jurídicamente reconocida como madre, no es la mujer que da a luz. En el caso de una inseminación con una donante o una fecundación in vitro con una donante, la mujer que da a luz no es la madre biológica. La gestación subrogada refuerza el abandono jurídico del vínculo biológico mencionado en el artículo 30 del Código Civil español.

La gestación subrogada replantea definitivamente el sentido de la relación entre lo biológico y lo jurídico. En efecto, la "ley humana” y la "ley de la naturaleza” se enfrentan a 
la reivindicación de un "derecho a tener hijas o hijos" que se sitúa fuera de la relación entre la procreación y la sexualidad. En efecto, la filiación jurídica constituye ahora un sistema normativo complejo construido sobre la base de un modelo que combinaba la Naturaleza y la voluntad. La procreación, hecho biológico, es una condición pero ya no sine qua non a la cual se añaden la voluntad y la realidad social y afectiva.

El Derecho español no acepta cualquier división del concepto de la maternidad y, de momento, rechaza la consagración jurídica de la maternidad de la mujer que tiene en gestación al niño o a la niña por cuenta de otra mujer. Probablemente, la razón del bloqueo jurídico frente a esta técnica de procreación radica en que, en realidad, la división "madre gestante madre genética" es provisional y anunciadora, tarde o temprano, del paso de la última frontera, la de la gestación fuera del cuerpo de la madre (ectogénesis) con la ayuda de una máquina que permitirá el desarrollo del feto fuera del útero: el útero artificial (ATLAN, 2005).

En el marco del principio de una igual filiación biológica materna y paterna, la cuestión de la filiación se simplificaría porque el útero artificial no tiene el mismo potencial transgresor que la gestación subrogada. Refuerza la igualdad entre la filiación materna y la filiación paterna. Si la gestación puede realizarse con una incubadora, las mujeres sin útero o con un útero no funcional pueden prescindirse de los servicios de una madre sustituta o la adopción.

La gestación subrogada sería inútil para estas mujeres infértiles, así como para las parejas homosexuales, siempre que se les otorgue la autorización - gracias a una donación de ovocitos o una recepción de embriones - de obtener un niño o una niña por ectogénesis sin pasar por el cuerpo de una mujer. Así pues, la paternidad gay se vería facilitada y desaparecería así el desequilibrio entre la maternidad lesbiana y la paternidad gay, siendo actualmente la primera más fácil de acceder que la segunda.

Por lo tanto, el útero artificial es compatible con un ajuste de la concepción de la maternidad. Por supuesto, las solteras y los solteros podrían usar la ectogénesis con una donación de gametos (del sexo ausente) o un huésped embrionario, pero para las mujeres esta posibilidad de dar a luz sola ya existe con técnicas actuales de reproducción asistida.

En definitiva, si el útero artificial no reemplaza las donaciones de gametos y, por lo tanto, no resuelve la cuestión del papel del tercero en la filiación y el acceso a los orígenes, permitiría abandonar la gestación subrogada que conlleva el riesgo de explotación de los cuerpos de las mujeres, así como la necesidad de reconocimiento del papel de la mujer gestante al lado de la madre.

También podemos considerar lo contrario, que la generalización del útero artificial va de la mano de un vínculo materno independiente de cualquier vínculo gestacional, incluso genético. Por una parte, la ectogénesis permitiría la desacralización de la maternidad en beneficio del vínculo materno, entendido como el cuidado de los niños y las niñas, sea el que

7 La experimentación en materia de reproducción humana permite no sólo romper el vínculo entre la sexualidad y la procreación sino también, en futuro no muy lejano, podría profundizar esta ruptura por la transgresión del carácter sexuado de la reproducción al negar la dualidad hombre-mujer. Un embrión podría estar creado a partir del patrimonio genético de dos mujeres. 
sea su sexo, puesto que ni la mujer ni el hombre tendrá a la prole en su cuerpo respectivo. Por otra parte, no hay nada que nos impida salir de la filiación, que haya o no donación de gametos, en la medida en que, debido a la desnaturalización de la procreación, el deseo de tener a un hijo o una hija tiene prioridad sobre la dimensión biológica del vínculo materno.

En los años setenta, algunos autores imaginaron que la crianza de las niñas y los niños, después de su incubación, tenía que ser compartida entre varios adultos en el marco de las familias comunitarias para evitar la reproducción del patriarcado generado por la familia nuclear (FIRESTONE, 1979). Hoy en día, esta idea sobrevive en las discusiones relativas a la "crianza compartida" en el seno de las familias homoparentales.

No obstante, en estos mismos debates, el significado del útero artificial en relación con el vínculo materno depende de las intenciones de su utilización y, por lo tanto, de los valores que prevalecen. Por el momento, la ectogénesis aparece más como una alternativa a la gestación subrogada que el medio para promover una maternidad múltiple basada en la voluntad y separada de cualquier referencia al vínculo biogenético. Sin embargo, el número creciente de países que legalizan la gestación subrogada parece indicar que, tarde o temprano, éstos adoptarán la técnica del útero artificial como método "normalizado" de procreación.

\section{CONCLUSIONES}

Consideraciones como la intención y la afectividad serán los elementos determinantes para construir esa nueva maternidad cuyo criterio biológico ya no es relevante. Así pues, la gestación subrogada - paso previo a la técnica del útero artificial - y el legislador que la regulará, neutralizarán a la vez el poder de la mujer sobre la reproducción y los inconvenientes del embarazo para la mujer que quiere tener una hija o un hijo.

Las tecnicas de reproduccion y de procreacion han contribuido a la existencia de varias figuras de maternidad posibles: la genética, la uterina y la intencional. Una dificultad mayor radica en el hecho de que nuestra sociedad no encuentra representaciones efectivas de esas figuras de madre.

Con la gestación subrogada, asistimos a un desplazamiento de la maternidad del ventre hacia una maternidad nutridora basada en la intención o la voluntad. La futura mecanización de la gestación por medio del útero artificial es ficticia, pero no por mucho tiempo. El Derecho tratará de regular esta técnica al igual que la gestación subrogada, cortando definitivamente el vínculo biológico en la maternidad. La maternidad será redefinida en otros términos que los de la Naturaleza.

\section{REFERÊNCIAS}

AGACINSKI, Sylviane. Corps en miettes. París: Flammarion, 2013. 
ATLAN, Henri. L'utérus artificiel. París: Le Seuil, 2005.

BAUDOIN, Jean-Louis; LABRUSSE-RIOU, Catherine. Produire l'homme, de quel droit? París: PUF, 1987.

CANTO-SPERBER, Monique; FRYDMAN, René. Naissance et liberté. La procréation. Quelles limites?. París: Plon, 2008.

ESPANA. Ministerio de la Presidencia, Relaciones con Las Cortes y Memoria Democrática. Real Decreto de 24 de julio de 1889 por el que se publica el Código Civil. Gaceta de Madrid, n. 206, 25 jul. 1889.

FIRESTONE, Shulamith. The Dialectic of Sex: the case for feminist revolution. Nueva York: Women's Press, 1979.

FONTY, Bernard; HUGUENIN, François. Les pères n’ont rien à faire dans la maternité. París: First Editions, 2003.

GIAMI, Alain; PY, Buno (dir.). Droits de l'homme et la sexualité. Vers la notion des droits sexuels? París: Archives de Etudes contamporaines, 2019.

GUERRINA, R. Mothering the Union: Gender Politics in the EU. Manchester: Manchester University Press, 2005.

LEMOULAND, Jean-Jacques. Droit de la famille, Couple et parenté. París: Ellipses, 2004.

MARRADES PUIG, Ana I. Luces y sombras del derecho a la maternidad: análisis jurídico de su reconocimiento. València: Universitat de Vàlencia, 2002.

MIGUEL ÁLVAREZ, Ana de. La revolución sexual de los sesenta: una reflexión crítica de su deriva patriarcal. Investigaciones feministas: papeles de estudios de mujeres, feministas y de género, n. 6, p. 20-38, 2015.

ROWLAND, Rober J. Los regímenes demográficos y sus contextos. Revista de Demografía Histórica, v. 33, n. 2, p. 185-224, 2015. 